\section{ESTADIO MUNICIPAL DE RIAZOR/LA CORUÑA}

\author{
Antonio Desmonts \\ Dr. Arquitecto
}

\section{Manuel Segura Ortiz}

Ingeniero de Caminos

Dragados y Construcciones, S. A.

EN el año 1979 el pleno del Excmo. Ayunta- miento de La Coruña encargó la redacción de un proyecto de remodelación del estadio municipal, de tal forma que sirviera para albergar una de las sedes del campeonato mundial del presente aก̃o.
El problema era complejo en si, ya que era necesario conseguir como mínimo 35.000 espectadores de aforo, respetar las pistas de atletismo ubicadas en el mismo estadio y adaptarse a las limitaciones físicas que imponia el palacio de los deportes (frontón, piscina cubierta y pistas polideportivas) consiguiendo al mismo tiempo un conjunto armónico que respetara el trazado en herradura que en su origen presentaba el estadio municipal de Riazor, volcado al mar a través de una gran arcada, que era su entrada principal y donde hoy se sitúa el palacio de los deportes.

El diseño arquitectónico se realizó olvidándose de todo y primando la idea central de La Coruña como ciudad marinera, lo cual se materializó en el diseño de las cubiertas de las distintas gradas, tribuna, marathon y preferencia.

Este diseño llevó a la concepción de una cubierta colgada, como la vela de un barco, de sus correspondientes mástiles y obenques.

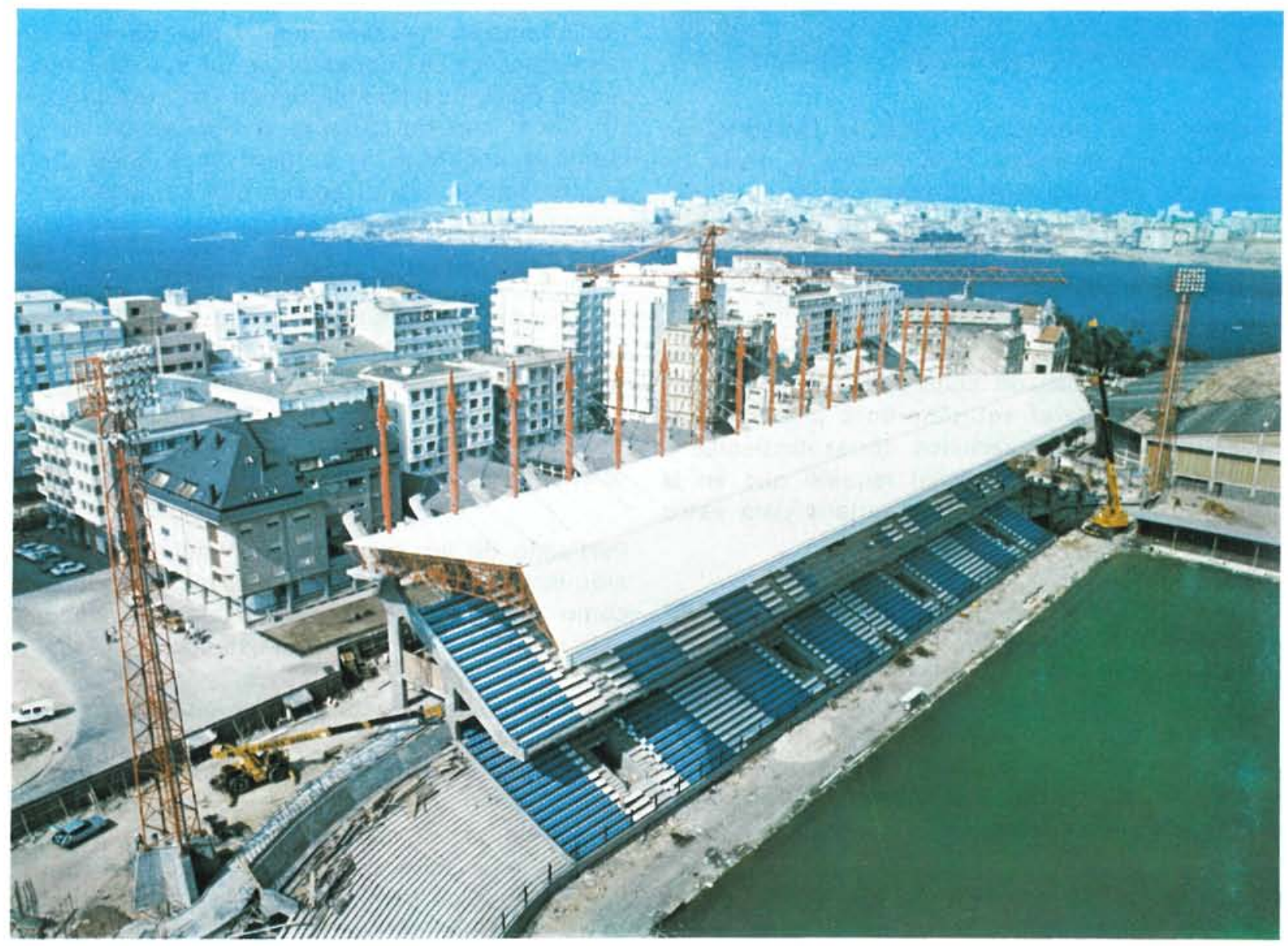



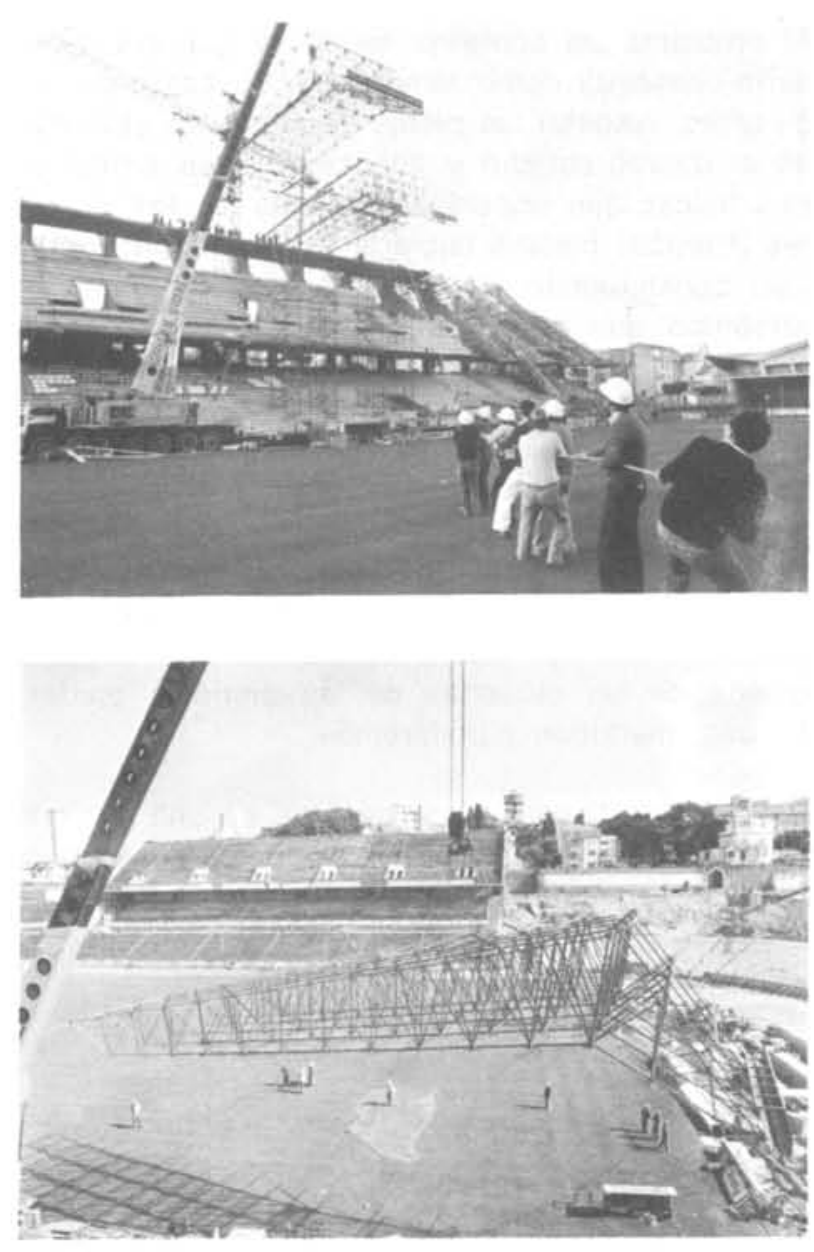

Aunque en el proyecto original la remodelación afectara exclusivamente a la tribuna y grada de marathon, defectos estructurales graves en la tribuna de preferencia obligaron a su demolición y posterior reconstrucción, lo cual ha supuesto la remodelación total del estadio.

Arquitectónicamente los problemas planteados radicaban en, además de conseguir las localidades previstas, alojar el equipamiento propio de la sede, asi como los servicios correspondientes a un campo de fútbol, lo cual requirió que en la tribuna principal se alojara un sótano para estos equipamientos.

En la tribuna principal el campo crea un porche cubierto a nivel de cota 0 que sirve como paseo integrado en el conjunto urbano.

Los accesos a la tribuna principal se realizan, a través de rampas, a la grada de marathon desde la calle, lo mismo que a la tribuna de preferencia actualmente remodelada.

Dentro de toda la remodelación sólo se ha conservado del antiguo campo municipal la torre de ma- rathon por razones sentimentales, de sobra conocidas por todos los coruñeses.

En todo momento se han buscado cómodos accesos a las localidades, una gran visibilidad y comodidad de todos los que presencien los partidos que se jueguen en esta sede y dotar a la ciudad de La Coruña de un estadio moderno.

La concepción estructural que incluia el proyecto de arquitectura era una solución integral de hormigón armado, donde únicamente las cubiertas espaciales y sus elementos de sustentación eran metálicos, recordando como ya dijimos anteriormente las velas de un barco. Fundamentalmente la estructura debia ser el elemento primordial de decoración de la obra y, por lo tanto, se pensó en hormigón visto, dada la tradición que Galicia presenta en cuanto a encofradores de alta cualificación, considerándose únicamente como elementos prefabricados las distintas gradas que componen las localidades del estadio. En todo momento, por lo tanto, la estructura, además de responder a sus necesidades resistentes, debia de ser un elemento estético e integrado en el conjunto, consideración ésta que imponia unas ciertas limitaciones de forma y dimensión.

Por último, es necesario indicar que las estructuras espaciales se cubrieron con un poliéster translúcido dotado de iluminación cenital, para producir un efecto especial durante la celebración de encuentros nocturnos $y$, al permitir el paso de la luz, reducir el natural agobio que producen en el espectador las marquesinas.

La iluminación del conjunto se realizó a base de una banda corrida sobre la marquesina de la tribuna principal y cuatro torres laterales, consiguiéndose con este conjunto un nivel de iluminación de 1500 lux sobre plano horizontal, valor óptimo para una perfecta retransmisión a través de televisión en color.

Partiendo de las premisas que impuso el diseño arquitectónico anteriormente expuesto y tomando como base el proyecto de subasta, se remodelo éste para conseguir el efecto estético.

Las soluciones estructurales que se han manejado en todo el estadio, son pórticos de hormigón armado arriostrados, bien de forjado birreticular en la tribuna principal y en la grada de marathon, a través del paseo de circunvalación que une por detrás de la torre de marathon ambas tribunas, bien de losa de hormigón armado prefabricada en la grada de preferencia. 
Los apoyos de la estructura espacial sobre la estructura de hormigón se han resuelto de forma distinta para cada una de las zonas romodeladas, utilizándose una losa continua en el caso de la tribuna principal, elementos aislados en forma de seta como capiteles de pilares en la grada de marathon, y una solución mixta para la grada de preferencia, compuesta por setas en forma de capiteles y una losa continúa de menor anchura.

En todo momento, tanto en la grada de preferencia como en la tribuna principal, se han dispuesto unos elementos de anclaje que sirven para sustentar los canalones vistos que recogen las aguas de cubierta y que. además, cumplen la función de proteger a los espectadores del viento.

El conjunto de la estructura de hormigón se diseñó partiendo de acero de alta resistencia $(5.000$ $\mathrm{k} / \mathrm{cm}^{2}$ de limite elástico) y con hormigón de alta capacidad, para evitar posibles problemas de oxidación por el ambiente en el cual se situa la obra y con una resistencia caracteristica de $200 \mathrm{k} / \mathrm{cm}^{2}$ a 28 dias.

El diseño de la obra de hormigón no entraña más problemas que los especificos de las solicitaciones impuestas por el uso del edificio y las caracteristicas geométricas de los diseños, consiguiéndose estructuras rígidas y con muy pequeñas deformaciones a carga vertical, lo cual da una gran sensación de confort al usuario, ya que sus deformaciones bajo carga total son muy inferiores a los limites admitidos por la normativa actualmente vigente.

El problema más complejo se presentó en el diseño del conjunto que forma la cubierta espacial, su apoyo en la estructura de hormigón y los elementos de anclaje, en hormigón armado, que integrados en las zancas de todas las tribunas remodeladas, sirven para absorber las solicitaciones que permiten el equilibrio de estos elementos. En la solución inicial, tanto en tribuna principal como en marathon, se fue a un mástil por pórtico, con sus correspondientes elementos de anclaje, mientras que en la grada de preferencia se modificó este criterio, disponiéndose un mástil y un elemento de anclaje por cada dos pórticos, para acentuar todavia más la sensación de esbeltez y de ligereza que en todo momento se quiso dar al diseño estructural de las marquesinas.

Conceptualmente todas las marquesinas se han diseñado en estructura espacial de tubo y bola, sobre esfera hueca que garantizara una perfecta ejecución de las uniones y que permitiera la vigilancia y supervisión de los cierres de apriete en
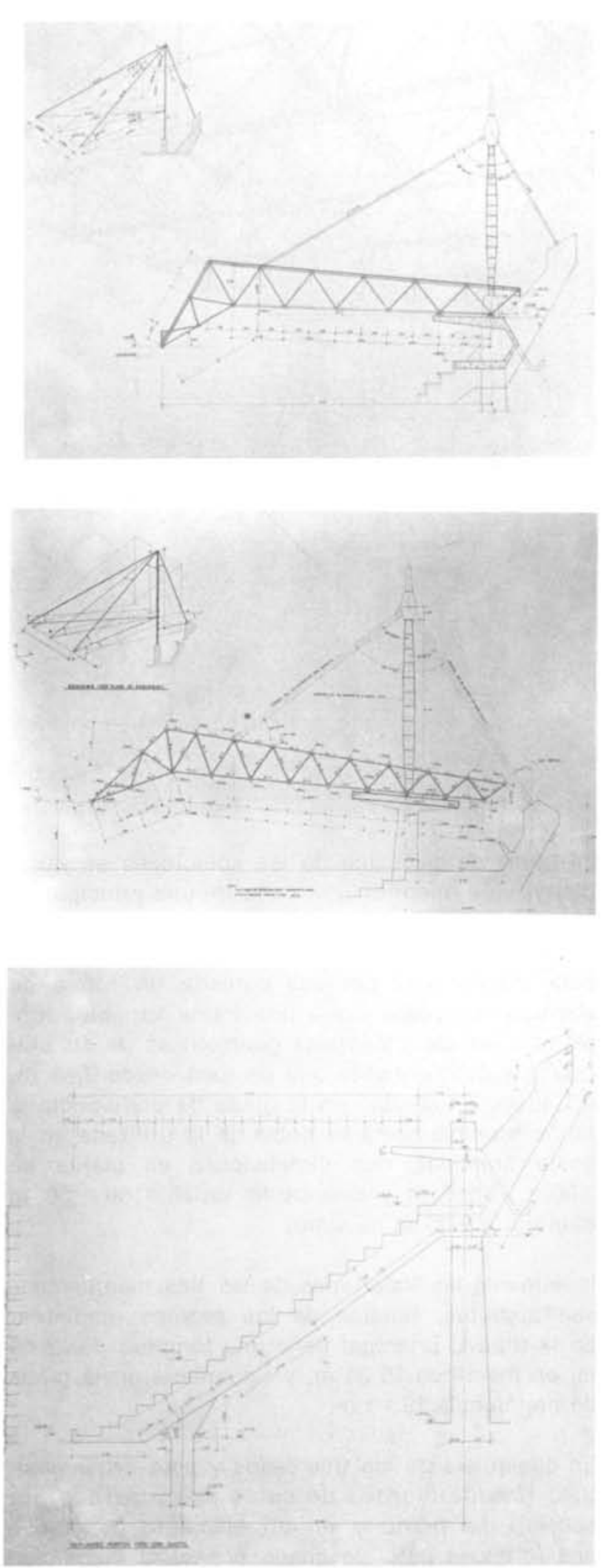

todos los tornillos de conexión. Todas estas condiciones nos llevaron a decantarnos por la solución que comercializaba en ese momento en España Perfrisa y cuyo diseño original pertenece al Arquitecto Pachi Alcalde. 


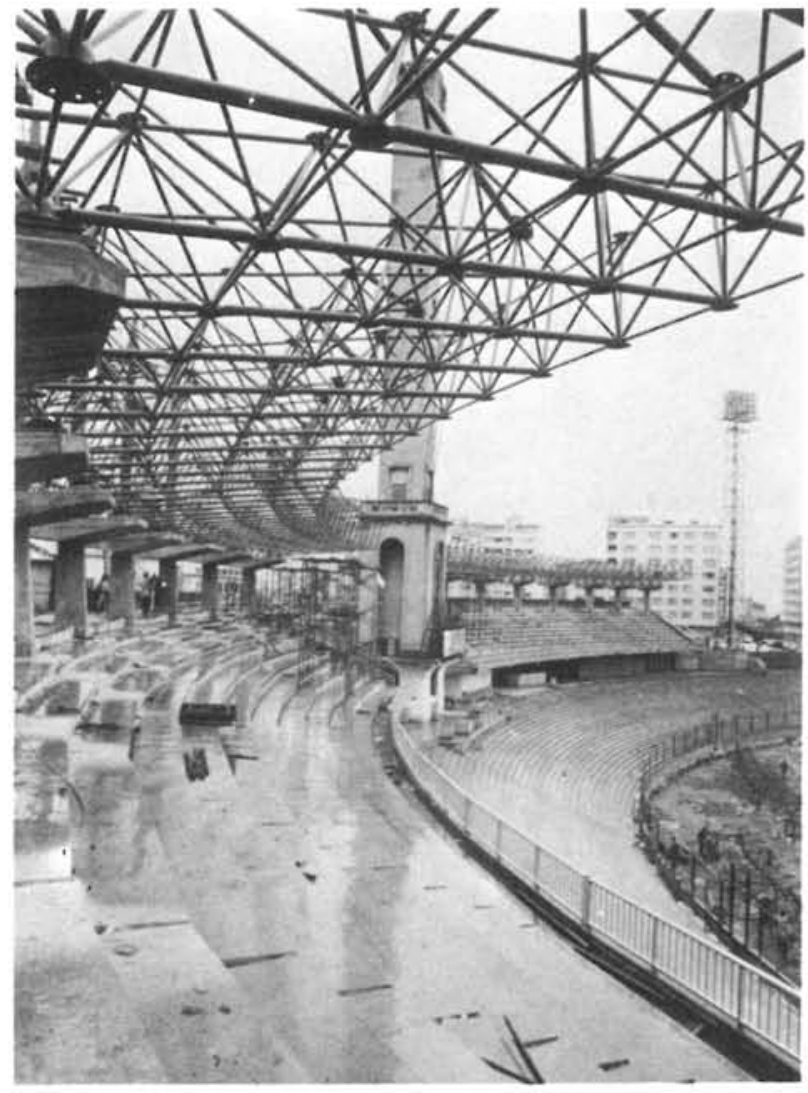

La trama de cada una de las soluciones es variable, ya que mientras que en la tribuna principal se fue a una trama básica de 1,7011 x 1,74 m, con canto variable (mínimo 1,70 $\mathrm{m}$, máximo 2,90 m) para marathon, al ser una cubierta en forma de abanico, nos plegamos a una trama variable, función de las características geométricas de su ubicación y con un canto que oscilara desde $0,60 \mathrm{~m}$, a 1,40 m. En cambio, en la grada de preferencia la trama sensiblemente es doble de la utilizada en la grada principal, con dimensiones en planta de $3,00 \times 3,7355 \mathrm{~m}$ y con canto variable de $1,50 \mathrm{~m}$ minimo a 2,30 m máximo.

Igualmente, las longitudes de las tres marquesinas son distintas, función de los propios graderíos. En la tribuna principal tiene una longitud de 23,40 $\mathrm{m}$, en marathon $15,30 \mathrm{~m}$, y finalmente, en la grada de preferencia, $19,10 \mathrm{~m}$.

En cualquiera de los tres casos y para evitar posibles levantamientos de estas marquesinas por succión de viento $y$ en un elemento decorativo que forma el peto de chapa prelacada en blanco con el cual se rematan todas las cubiertas, se aloja un contrapeso de hormigón in situ que realiza la misión anteriormente citada, y que en el caso concreto de la tribuna principal sirve además como apoyo de la banda de iluminación y como pasarela de inspección de la misma.
Todas las estructuras espaciales están lacadas en color rojo, con una pintura en base esmalte de poliéster que garantiza la conservación frente a la atmósfera marina de la ciudad de La Coruña.

Como ya se dijo anteriormente, estas soluciones están cubiertas con poliéster traslúcido en color blanco, para permitir el paso de la luz durante el dia y la iluminación del ambiente exterior durante la noche, a través de los correspondientes equipos de iluminación cenital de las propias marquesinas.

Su sustentación se materializó a través de mástiles apoyados en su base sobre rótulas esféricas, suministradas por Imbadelca, para cargas variables que oscilan desde 190 t a $35 \mathrm{t}$, según las distintas soluciones en función de las correspondientes marquesinas. Estas rótulas, que van apoyadas sobre la estructura de hormigón, reciben los mástiles de sección variable, de acero, que están coronados por una cabeza hueca, donde se alojan los anclajes de las barras dywidag, suministrados y montados por EYSA. Estas cabezas Ilevan cierres laterales atornillados colocados posteriormente a la ejecución de los anclajes y antes de llevar a efecto su correspondiente inyección.

Los mástiles igualmente tienen alturas variables en función de cada una de las soluciones. En concreto estas piezas presentan una dimensión máxima de $0,60 \times 0,30 \mathrm{~m}$ de sección para la tribuna principal, con una altura total de $12,60 \mathrm{~m}$; en el marathon tiene una sección de $0,40 \times 0,25 \mathrm{~m}$ en planta y una altura de $8 \mathrm{~m}$; y finalmente en la grada de preferencia, presenta la misma sección máxima en planta que en la tribuna principal, con una altura total de $12,50 \mathrm{~m}$.

Esta estructura fue fabricada y montada por VEMSA, S. A., y, previo chorro de arena al grado S3 de la norma sueca, fue pintada con un tratamiento similar al de la estructura espacial, con la prescripción básica de mantener la misma resistencia a la corrosión frente a ambientes marinos que la solución anteriormente citada. Su terminación fue en el mismo color rojo que el resto de la cubierta principal.

Las barras dywidag fueron protegidas del ambiente exterior mediante tubo de PVC rigido en color gris oscuro, que asemejaba a los elementos de sustentación de las velas marinas, con diámemetros entre 80 y $200 \mathrm{~mm}$, según los casos, que permitia además de su protección, la inyección de una lechada especial de mortero de cemento, inyectada a presion, que recubre la totalidad de las barras y garantiza la no corrosion de estos elementos. En todo momento, como se deduce de la 
explicación anterior, se culdaron y extremaron las precauciones para que la solución proyectada en el conjunto de marquesinas, mástlles y barras de sustentación luera resistente la corrosion mam rina y tuviera una conservación reducida, al mismo tiempo que simple, mediante inspecciones pe ribdicas que pusieran en evidencla la existencia o no de pequenos defectos que, al ser rápidamente subsanables, no produjeran problemas irremedia bles en el mencionado diseño.

Conceptualmente, por lo tanto, la malla espacial, de canto variable, presenta un trabajo predominante en la dirección transversal, mientras que en el sentido longitudinal de la misma unicamente cuenta con un elemento de repario, ya que la es. tructura, al disponer de apoyos localizados y sustentaciones ubicadas en zonas determinadas, debe componer como un elemento continuo en ese sentido.

La elección de la estructura espacial a base de bolas y barras se decidió por su estética al mismo llempo que por su reducido peso, lo cual acentúa la sensación de ligereza. Es necesario indicar que mientras que la marquesina de la tribuna principal pesa $20 \mathrm{~kg} / \mathrm{m2}$, la solucion de marathon ronda $10 \mathrm{~s}$ $10 \mathrm{~kg} / \mathrm{m} 2$ y la correspondiente a la grada de pre ferencla está en $14 \mathrm{~kg} / \mathrm{m} 2$. Este peso no se hubie ra conseguido con ninguna solución tradicional a base de estructura metalica, la cual además no hubiere presentado el mismo valor estético.

Un problema secundario tue la resolucion del anclaje de las barras dywidag en las proplas bolas de la estructura espacial, lo cual obligo a la utif. zación de elementos de grandes dimensiones $(200$ por $20 \mathrm{~mm}$ ), girados con respecto a su posición logica para asi garantizar el anclaje de las barras, su fácil acceso y la disposición de los elementos de sujeción del sistema elegido como sustentaw ción de la esiructura.

Las bolas de apoyo en la estructura de hormigón fueron elegidas basandose en las soluciones estándar de la firma comercial, ya que son puntos fjos, articulados, usuales en soluciones de esta naturaleza.

La forma especial de la cubierta que presenta inclinaciones variables de todos los elementos que componen la estructura espacial, obligo a desarro llar un programa automático de ordenador que diera no sólo las longiludes de las barras, sino también los ángulos de incidencla de los mismas en las bolas. Esle problema lue mucho más complejo en la tribuna de marathón, ya que no sólo presentaba la variabilidad de los ángulos de inclim nación de las diagonales, sino también la no constancla de las barras longitudinales que en cada caso eran secantes a circulos de radio variable.

Unicamente gracias al ordenador se pudo tener un replanteo concreto de estos ángulos que permitio una fabricación adecuada y la no existencia de problemas en el montaje de la estructura.

Como indice de rendimiento, la tribuna principal que liene en planta alrededor de $3.400 \mathrm{~m}^{2}$ y que se montaba a unos $35 \mathrm{~m}$, de altura, se ejeculó en 20 dias con un equipo de 16 hombres. En mara. thón, al no existir problemas de plazo y al ejecutarse en invierno con problemas atmostéricos, el tiempo ve más largo, mientras que la grada de preferencia se monto en un plazo de 15 dias con una supericie en planta de unos $2.500 \mathrm{~m}^{2}$ y con un equipo similar al de la cubierla de la tribuna principal.

Finamente debemos indicar que el anclaje por la estructura de hormigon de las barras dywidag, se realizo alojando un conducto en la citada estructu. ra, que permitiera el paso de las mencionadas ba. ras y que se anclara con los correspondientes mecanismos; los cuales veron protegidos de la corrosion mediante caparuzas de PVC en el mis. mo color que los tubos empleados en la protecclon de las barras.

Complementariamente, la ribuna principal y la grada de preterencla presentan en sus fachadas unos canalones vistos para recoger las aguas de las marquesinas, lo cual se resolvió con una solución mixta de hormigon y chapa, siendo la zona metalica una artesa de acero cuyos elementos interio res están pintados con el mismo material que el resto de los elementos metalicos de la estruc ura. La chapa interior es gavanizada, mientras que su limite externo es una chapa prelacada en color blanco. Estos elementos desaguan a través de bajantes vistas que se alojan en unos rehundim dos de los plares de hormigón. El conjunto del canalon, homigón y acero, es un elemento deco rativo en fachada que, además, cumple una misión funcional y que, al igual que toda la solución estructural del estadio, presenta un diseño que le confiere un aspecto integrado en el planteamiento estético de todo el contunto.

El proyecto de toda la estructura de hormigón se realizó mediante un programa mecanizado, sobre base ordenador IBM, y con un elemento de cálcu10 procedente del MIT linstivio Tecnologico de Massachusets). 

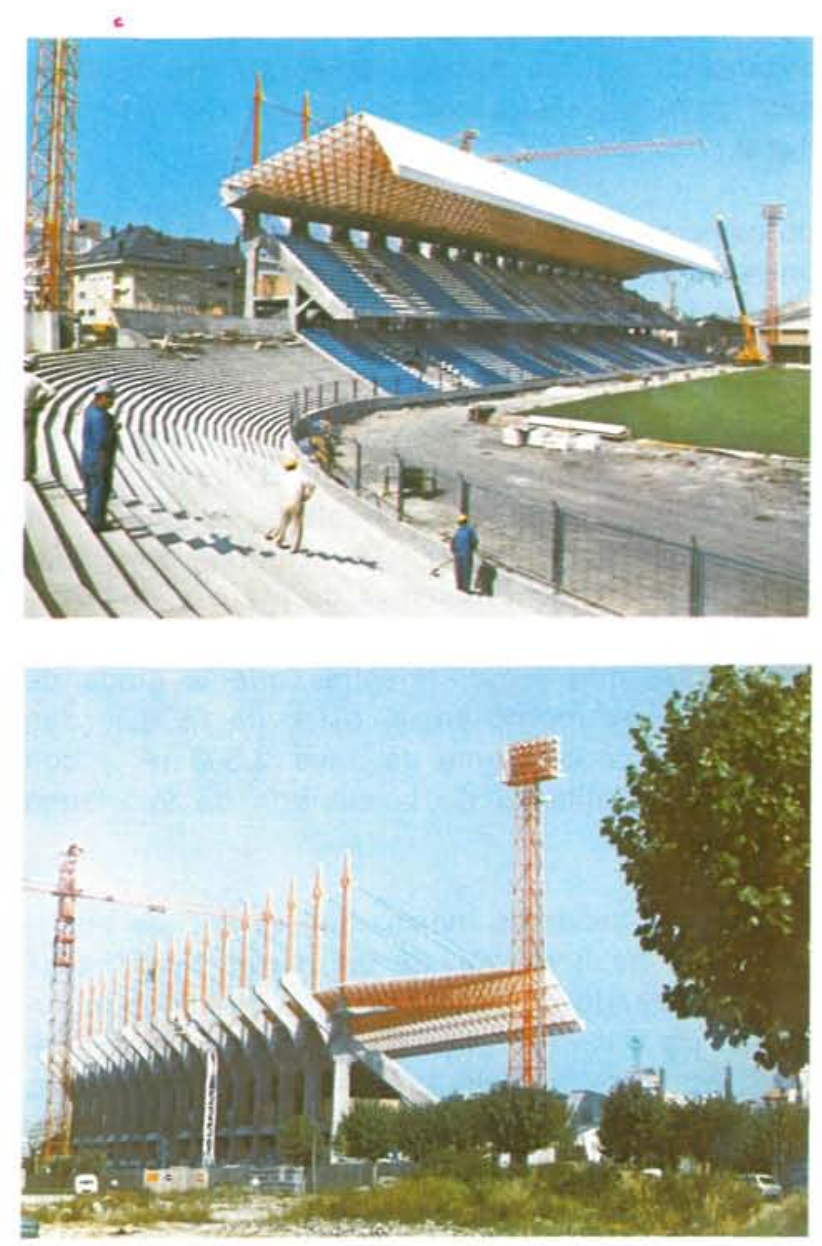

Unicamente deberemos de resaltar que la ejecución de la tribuna principal se efectuó en un plazo récord para ser inaugurada con el trofeo Teresa Herrera en agosto de 1981, habiéndose comenzado las obras en febrero del mismo año. La grada de preferencia se realizó en un plazo de dos meses y medio.
La ejecución de la obra de hormigón se realizó en base a encofrados industrializados, diedros para pilares en madera, dado el carácter visto de estos elementos, cimbras mixtas metálicas-madera, para todos los elementos lineales (pórticos) y encofrados industrializados para las soluciones de forjado birreticular con bovedillas de plástico recuperables suministradas por MILLS, S. A. Esta industrialización de los encofrados, unida a la alta cualificación de la mano de obra, que en Galicia es tradicional en los encofradores, permitió la calidad que presenta el aspecto externo de toda la obra de hormigón.

A lo largo de toda la ejecución, el control de calidad de la Administración, asi como el propio autocontrol de la empresa constructora, ha permitido garantizar la correcta ejecución de la estructura y el alcanzar en obra resistencias de hormigón muy superiores a las inicialmente estimadas en el diseño.

Finalmente es necesario recalcar que tanto la concepción como el diseño estructural ha intentado ( $y$, en nuestra opinión, en parte ha conseguido) ofrecer una solución de estructura adecuada $y$, en el caso concreto de las marquesinas, una solución original muy ligada al carácter marinero de la ciudad de La Coruña.

El conjunto del estadio una vez remodelado, presenta un aspecto francamente agradable, de fácil acceso y con una gran visibilidad en todos sus puntos, lo que permite la correcta visión no sólo de los partidos de fútbol, sino también de los acontecimientos deportivos que se puedan realizar en sus pistas de atletismo.

\section{* $* \quad *$}

